\title{
Assistência de enfermagem a pacientes portadores de insuficiência cardíaca descompensada: uma revisão integrativa
}

RESUMO | O objetivo do estudo foi identificar os cuidados de enfermagem a pacientes portadores de insuficiência cardíaca descompensada descritos em literatura nacional e internacional. Método- Foi realizada uma Revisão Integrativa. A busca foi realizada de forma sistematizada nas bases de dados LILACS, BDENF, IBECS, MEDLINE e Pubmed. Resultados- A amostra constituiuse de oito artigos, sendo possível identificar intervenções de enfermagem educativas para o controle e prevenção da doença, intervenções durante a internação e após a alta hospitalar. Conclusão- Dentre os estudos selecionados, ficou evidenciado que as intervenções de enfermagem são fundamentais para prevenção e promoção a saúde a pacientes portadores de insuficiência cardíaca descompensada nos diversos cenários de atenção à saúde. O acompanhamento e a educação em saúde ao paciente com insuficiência cardíaca têm grande potencial para atingir mudanças de atitudes e comportamento no enfrentamento a doença.

Palavras-chaves: Enfermagem; Insuficiência cardíaca; Cuidados de enfermagem.

ABSTRACT |The aim of the study was to identify nursing care for patients with decompensated heart failure described in national and international literature. Method- An Integrative Review was carried out. The search was carried out systematically in the databases LILACS, BDENF, IBECS, MEDLINE and Pubmed. Results- The sample consisted of eight articles, it was possible to identify educational nursing interventions for the control and prevention of the disease, interventions during hospitalization and after hospital discharge. Conclusion- Among the selected studies, it was evidenced that nursing interventions are essential for the prevention and promotion of health to patients with decompensated heart failure in the different health care scenarios. Monitoring and health education for heart failure patients has great potential to achieve changes in attitudes and behavior in coping with the disease.

Keywords: Nursing; Heart Failure; Nursing care.

RESUMEN | El objetivo del estudio fue identificar los cuidados de enfermería para pacientes con insuficiencia cardíaca descompensada descritos en la literatura nacional e internacional. Método- Se realizó una Revisión Integrativa. La búsqueda se realizó de forma sistemática en las bases de datos LILACS, BDENF, IBECS, MEDLINE y Pubmed. Resultados- La muestra estuvo formada por ocho artículos, fue posible identificar intervenciones educativas de enfermería para el control y prevención de la enfermedad, intervenciones durante la hospitalización y tras el alta hospitalaria. Conclusión- Entre los estudios seleccionados, se evidenció que las intervenciones de enfermería son fundamentales para la prevención y promoción de la salud de los pacientes con insuficiencia cardíaca descompensada en los diferentes escenarios asistenciales. El seguimiento y la educación sanitaria de los pacientes con insuficiencia cardíaca tiene un gran potencial para lograr cambios en las actitudes y el comportamiento para afrontar la enfermedad.

Palabras claves: Enfermería; Insuficiencia Cardíaca; Atención de Enfermería.

Flávia Mariana Mendes Diniz

Enfermeira do Hospital das Clínicas da Universidade Federal de Minas Gerais (HC-UFMG). Mestre Multiprofissional em Terapia Intensiva pelo Instituto Multidisciplinar Brasileiro de Educação em Saúde (IMBES). ORCID: 0000-0002-4684-7595

\section{Karla Cordeiro Gonçalves}

Enfermeira do HC-UFMG. Mestre em Ciências Aplicadas à Saúde do Adulto pela UFMG. Especialista em cardiologia pelo Hospital das Clínicas da Faculdade de Medicina da Universidade de São Paulo (HC-FMUSP).

ORCID: 0000-0002-5123-9308

Recebido em: 30/12/2020

Aprovado em: 26/01/2021

INTRODUÇÃO

$\Lambda$ Insuficiência cardíaca (IC) é conceituada como uma síndrome clínica complexa, onde o coração é incapaz de bombear sangue de forma a atender às necessidades metabólicas tissulares, ou pode fazê-lo somente com elevadas pressões de enchimento ${ }^{(1)}$.

Segundo estimativas, a IC afeta em torno de 26 milhões de pessoas em todo o mundo, sendo no Brasil, a principal causa de internação por motivos cardiovasculares com uma taxa de $21 \%$ dos $\operatorname{casos}^{(2)}$.

Ainda no Brasil, quando se fala em pacientes acima de 60 anos, a IC descompensada constitui a primeira causa de internação hospitalar, sendo responsável por alta taxa de hospitalização e alta taxa de mortalidade, caracterizando-se como uma doença de alta prevalência. Seu desenvolvimento é comum como desfecho da maioria das doenças cardíacas, sendo ela um grande desafio na abordagem medica multiprofissional ${ }^{(3)}$. No mundo a IC representa um grande problema de saúde pública ${ }^{(3)}$.

Sendo uma doença com alta taxa de internação, se torna fundamental acompanhar a doença em todos os seus estágios, desde medidas de controle e promoção da saúde até intervenções especificas durante a descompensação. $\mathrm{O}$ enfermeiro tem grande papel nessa abordagem, podendo ser facilitador em todos os níveis de saúde, tendo as inter- 
venções de enfermagem o propósito de atender satisfatoriamente as necessidades do paciente ${ }^{(4,5)}$.

A gestão e o acompanhamento do paciente portador de IC pela equipe multiprofissional é fundamental. Dentre esses, o enfermeiro é tido como um agente de destaque no potencial de reduzir a morbimortalidade por IC. Sua abordagem tem caráter amplo, desde a educação em saúde na adesão ao tratamento, farmacológico e não-farmacológico, a fim de evitar complicações do quadro, até intervenções durante a internação por descompensação ${ }^{(5)}$.

Considerando que a IC é uma síndrome de elevada prevalência e com alta taxa de descompensação, o objetivo deste estudo é identificar quais os cuidados de enfermagem a pacientes portadores de insuficiência cardíaca descompensada descritos em literatura nacional e internacional.

\section{MÉTODO}

Trata-se de uma Revisão Integrativa, onde permite sintetizar vários tipos de estudos para análise de amplo conhecimento e síntese de determinado assunto ${ }^{(6)}$.

A revisão foi realizada em 6 etapas, conforme Mendes, Silveira e Galvão ${ }^{(7)}$ : 1) Identificação do tema e seleção da hipótese ou questão de pesquisa para a elaboração da revisão integrativa; 2) Estabelecimento de critérios para inclusão e exclusão de estudos/amostragem ou pesquisa de literatura; 3) Definição das informações a serem extraídas dos estudos selecionados/categorização dos estudos; 4) Avaliação dos estudos incluídos na revisão integrativa; 5) Interpretação dos resultados e, 6) Apresentação da revisão/ síntese do conhecimento.

A questão norteadora da pesquisa foi elaborada utilizando o acrônimo PICO, P (População ou Problema: Portadores de IC), I (Intervenção: assistência de enfermagem), C (Comparação ou Controle: Não se aplica a esse estudo), O (Outcomes ou Desfecho ou Resultado: Inter- venções de enfermagem) ${ }^{(6)}$, resultando na seguinte questão norteadora: Quais intervenções de enfermagem podem ser aplicadas aos pacientes portadores de insuficiência cardíaca descompensada?

Foi realizado consulta ao Descritores

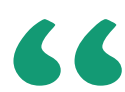

\section{Considerando} que a IC é uma síndrome de elevada prevalência e com alta taxa de descompensação, o objetivo deste estudo é identificar quais os cuidados de enfermagem a pacientes portadores de insuficiência cardíaca descompensada descritos em literatura nacional e internacional.
Ciência da Saúde (DeCS) e ao MeSHTerms (Medical Subject Headings) da PubMed, a fim de selecionar os descritores em português, inglês e espanhol a serem utilizados na busca. Os identificados foram: "insuficiência cardíaca", "Heart Failure", "Insuficiencia Cardíaca", "Cuidados de Enfermagem", "Nursing Care" e "Atención de Enfermería".

Os critérios de inclusão foram artigos científicos publicados nos últimos 10 anos, como forma de acompanhar a evolução da assistência prestada a pacientes com insuficiência cardíaca descompensada e apresentar o mais atual da literatura. E, trabalhos escritos em português, inglês e espanhol. Como critério de exclusão foram definidos os artigos de revisão e estudos que não responderam à pergunta norteadora e artigos duplicados. Os artigos encontrados em duplicidade foram contabilizados na base de dados com maior número de referências. Para a busca foi realizado o cruzamento dos descritores utilizando-se da lógica booleana no campo de busca.

A busca da literatura foi realizada no mês de agosto de 2020, por meio de dois portais de pesquisa: 1) BVS em que foram realizadas as buscas nas bases de dados: Literatura Latino-Americana e do Caribe em Ciências da Saúde (LILACS), Base de Dados de Enfermagem (BDENF) e Índice Bibliográfico Espanhol de Ciências da Saúde (IBECS). 2) PubMed em que foi realizada a busca de literatura na base de dados Medical Literature Analysis and Tetrieval System Online (MEDLINE). A validação da revisão ocorreu por meio de dois pesquisadores no intuito de aumentar a confiabilidade do estudo.

A coleta de informações foi realizada com o auxílio de um instrumento elaborado de acordo com o objetivo deste estudo. O instrumento contém informações relacionadas à título da publicação, autores, ano, objetivos, amostra e descrição dos cuidados de enfermagem aos pacientes portadores de insuficiência cardíaca. Os estudos selecionados foram classificados quanto ao nível de evidência ${ }^{(8)}$. 
DISCUSSÃO

A combinação dos descritores resultou em 460 publicações. Após aplicação dos critérios de inclusão mantiveram-se 263 publicações. Pela leitura dos títulos e resumos, foi possível excluir aqueles estudos que não abordaram o tema proposto. Desses, foram selecionados 30 artigos para leitura na íntegra, sendo que nove artigos responderam à questão norteadora e constituíram a amostra final desta revisão (Figura 1).

Verificou-se que $87,5 \%$ dos artigos escolhidos foram encontrados através do portal de pesquisa BVS e 12,5\% na PubMed. Em relação ao ano de publicação, $50 \%$ dos trabalhos foram publicados nos últimos 5 anos.

No que tange ao idioma, os artigos publicados em português compreendem $25 \%$, em inglês também: $25 \%$ e publicados nos dois idiomas, português e inglês $50 \%$. Em relação ao nível de evidência dos estudos, observou-se que $50 \%$ dos artigos são classificados no nível quatro, $37,5 \%$ no nível cinco e 12,5\% no nível dois.

\section{Figura 1: Percurso para busca e seleção dos estudos nas bases de dados.}

Combinação de descritores (Português, inglês e espanhol) 460 publicações

460 publicações

Aplicação dos critérios de inclusão

\begin{tabular}{l|l}
\hline Excluídos: 197 publicações & Excluídos: 197 publicações \\
& $\checkmark$
\end{tabular}

Aplicação dos critério de exclusão na leitura do título e resumo Excluídos: 233 publicações Restante: 30 publicações

Aplicação do critério de exclusão na leitura na íntegra Excluídos: 22 publicações risco da descompensação da IC são suscetíveis a intervenções de educação em saúde, que com o tempo reduzem a chance de internações e/ou morte prematura pela IC ${ }^{(11)}$.

Dentre as estratégias educativas, existem diversas formas de intervir como encontros educativos, utilização de materiais educativos impressos, abordagem através de visita domiciliar e até acompanhamento telefônico. Essas intervenções não apenas melhoram o conhecimento da IC pelo paciente como também estimulam o pensamento crítico reflexivo e a autogestão do processo saúde-doença ${ }^{(11)}$.

Nesse sentido, as intervenções e os cuidados de enfermagem ao paciente de IC tem por objetivo melhorar a resistência e capacidade de seu organismo, estabelecendo metas conjuntas e observando medidas farmacológicas e não farmacológicas ${ }^{(10)}$.

Além da abordagem farmacológica ${ }^{(12)}$, há comprovação de que o tratamento não farmacológico tem grande papel na prevenção e controle da IC ${ }^{(10)}$.

Dentre as ações relacionadas a intervenções e cuidados não farmacológicos estão orientações de suporte nutricional especialmente voltadas à restrição de sódio e líquidos, controle e monitoramento de peso, estímulo à vida ativa com exercícios físicos, técnicas de melhoramento do sono e repouso, e especialmente o reconhecimento dos sinais e sintomas de descompensação clínica ${ }^{(9 ; 12)}$.

Intervenções de enfermagem durante a internação

Os pacientes de IC quando admitidos na emergência, exigem grande preparo da equipe, é necessária rápida avaliação para definição de condutas adequadas e baseadas nas melhores evidências científicas. Nesse momento o exame físico se mostra uma grande ferramenta para diagnóstico e manejo da IC descompensada ${ }^{(10)}$.

Em uma abordagem inicial, deve-se priorizar medidas de suporte básico e 
avançado de vida como forma de preservação da vida do paciente. A equipe de enfermagem deve estar pronta para atender demandas emergenciais ${ }^{(10)}$.

Com o paciente já estabilizado, observa-se a avaliação da perfusão periférica como uma das principais intervenções de enfermagem, a fim de prevenir o desenvolvimento de tromboembolismo, além de acompanhar a função respiratória através a observação da cianose ${ }^{(10 ; 13 ; 14)}$.

Também relacionados a função respiratória, se destacam outras intervenções como a manutenção do posicionamento no leito mantendo conforto do paciente com cabeceira a pelo menos $30^{\circ}$, que facilita a respiração e diminui o retorno venoso ao coração, aliviando congestão pulmonar, além da realização da ausculta e controle hídrico para investigação de sinais de congestão ${ }^{(10 ; 13 ; 15)}$.

Nos pacientes congestos, foram levantadas algumas intervenções importantes como avaliação diária de edema, realização controle hídrico, administração de diuréticos prescritos, observação da diurese. Alguns sinais específicos de sobrecarga hídrica devem ser observados como, turgência jugular, dispneia, ganho rápido de peso e ruídos adventícios pulmonares ${ }^{(9 ; 10 ; 14 ; 15)}$.

Alguns desses pacientes são submetidos ao uso da oxigenoterapia, tendo o enfermeiro um papel fundamental em sua administração. Dentre as intervenções, estão: manutenção do nível da água no umidificador, identificação de sinais de hipóxia cerebral e monitoramento da frequência respiratória ${ }^{(10 ; 13 ; 14 ; 15 ;)}$.

Destacam-se ainda intervenções que objetivam a prevenção de aspiração e lesões de pele como posicionamento do paciente em Fowler e mudanças de decúbito em intervalos regulares. Essas medidas focam na segurança, ao prevenir riscos potenciais à saúde desse paciente ${ }^{(10 ; 13 ; 14)}$.

Se torna importante também estimular a mobilidade a fim de diminuir perdas funcionais pela descompensação da doença, deve-se estimular a deambulação levando em consideração os limites de capacida- de do paciente e como facilitador deve-se providenciar cama baixa, encorajar a sentar-se à beira do leito conforme a tolerância e auxiliar o paciente a transferir-se, conforme a necessidade ${ }^{(13 ; 15 ; 16)}$
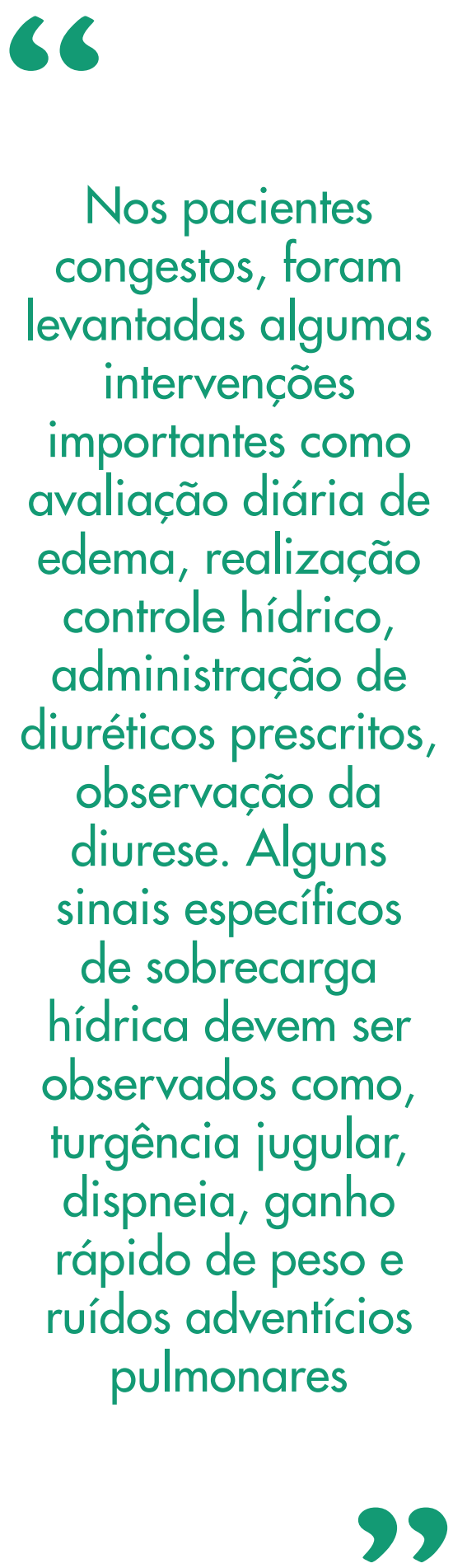

Outro problema relativamente comum em pacientes internados por IC descompensada é a presença de dor, algumas intervenções do enfermeiro são: avaliar o nível de dor, os fatores que intensificam e aliviam o sintoma; administrar analgésicos aprazados conforme o quadro do paciente e investigar a resposta à medicação para o alívio da dor, além de auxiliar em medidas de conforto para melhora indireta da dor como adotar medidas de controle do ambiente para melhora da qualidade do sono e reduzam a ansiedade ${ }^{(13 ; 14 ; 15)}$.

Essas intervenções podem ser realizadas pelo próprio enfermeiro ou prescritas para realização do técnico sob supervisão. É importante que todas as prescrições e orientações sejam facilmente compreendidas pela equipe de enfermagem. Cabe ao enfermeiro a definição as intervenções e priorização das atividades baseadas em evidências científicas para melhor atendimento ao paciente ${ }^{(14)}$.

Intervenções de enfermagem para alta hospitalar

A educação em saúde é potencial fortalecedor para o conhecimento da própria enfermidade pelo paciente, assim como a relação entre os medicamentos, os hábitos de vida e a doença. As Intervenções educativas de enfermagem realizadas durante a internação hospitalar para a alta trazem melhora do conhecimento da IC, do autocuidado e da qualidade de vida para pacientes e seus familiares. Esse conhecimento pode alterar os índices de re-internação ${ }^{(10)}$.

O conhecimento do enfermeiro sobre a terapia medicamentosa é a base de um dos principais cuidados de enfermagem no preparo do paciente para a alta, as orientações farmacológicas. Essas orientações devem ser realizadas de acordo com a prescrição específica proposta a esse paciente, reafirmando junto ao paciente o nome correto das medicações, a dose adequada, o horário regular e a via correta de administração destas e suas principais indicações, 
reforçando, sempre que necessário, os possíveis efeitos adversos e colaterais que podem ocorrer ${ }^{(9 ; 12)}$.

Além das intervenções voltadas as orientações sobre a terapia farmacológica, foi identificada também a importância do reconhecimento dos principais sinais e sintomas da IC, especialmente os sinais de piora como a dor ou desconforto precordial, dispneia, ortopneia, fadiga e edema de membros inferiores ${ }^{(12)}$.

Além disso, destacam-se também informações sobre medidas de controle da IC, como melhora dos hábitos alimentares, controle da ingesta hídrica, e preocupação com o estado nutricional. As orientações amplas dos fatores precipitantes podem potencializar os efeitos do tratamento ${ }^{(12 ; 15)}$.

Reafirmando a importância dessas ações pela equipe de enfermagem, a educação em saúde e o planejamento de alta se mostram ferramentas cada dia mais uteis para auxílio do controle de sintomas, e tem grande potencial para redu-

“

ção do número de reinternações por IC descompensada ${ }^{(12)}$

\section{CONCLUSÃO}

\section{Este trabalho permitiu elencar as principais intervenções realizadas em} diversos cenários, como o ambiente domiciliar e o hospitalar.
Os estudos apresentam as intervenções de enfermagem como fundamentais para prevenção e promoção da saúde a pacientes portadores de insuficiência cardíaca, sendo essas intervenções, parte de um acompanhamento individualizado que deve levar consideração as características especificas de cada paciente, promovendo assim o autocuidado e a auto responsabilização.

Este trabalho permitiu elencar as principais intervenções realizadas em diversos cenários, como o ambiente domiciliar e o hospitalar.

O acompanhamento e a educação em saúde ao paciente com IC utilizadas nos diversos níveis de atenção à saúde, tem grande potencial de atingir mudanças de atitudes e comportamento no enfrentamento a doença.

\section{Referências}

1. Wacker $P$, Nunes $P V$, Forlenza 0 . Delirium: uma perspectiva histórica. Rev Psiquiatr Clín. 2018; 32 (3): 97-103.

2. Bastos AS, Beccaria LM, Silva DC, Barbosa TP. Identificação de delirium e delirium subsindromático em pacientes de terapia intensiva. Rev Bras Enf. 2019; 72 (2): 463-467..

3. Bisinotto FMB, Silveira LAM, Silva RO, Martins LB. Delirium pós-operatório no idoso. Onde estamos?. Rev Med Minas Gerais. 2017; 27 Supl 2: S52-S66. 4. Lourenço BC, Narciso AC, Amorim EH, Almeida FCA, Cruz RAO. Processo do cuidar em enfermagem no perioperatório de cirurgia cardíaca. Braz. J. Surg. Clin. Res. 2013; 22: 2858-2866.

5. Neves AFC, Giustina KPD. Estudo sobre delirium em pós-operatório de cirurgia cardíaca, em um hospital de alta complexidade da região sul de Santa Catarina [monografia disponível da internet]. [Santa Catarina] UNIEDU; 2014 [Acesso em: 06 set. 2020]. Disponível em: http://www.uniedu.sed.sc.gov.br/ wp-content/uploads/2014/04/amanda_fernandes_candido_neves.pdf.

6. Oliveira FRA. Incidência, fatores preditores e consequências do delirium no pós operatório de cirurgia cardíaca [Tese disponível na internet]. [São Paulo] Faculdade de Medicina da universidade de São Paulo; 2015 [Acesso em: 05 set. 2020]. Disponivel em: https://www.teses.usp.br/teses/disponiveis/5/5131/ tde-11092015-160812/pt-br.php.

7. Souza MT, Silva MD, Carvalho R. Revisão integrativa: o que é e como fazer. Einstein. 2010; 8 (1): 102-106.

8. Santos CMC, Pimenta CAM, Nobre, MRC. A estratégia pico para a construção da pergunta de pesquisa e busca de evidências. Rev Latino-am Enfermage. 2007 mai; 15(3).

9.Ursi ES, Galvão CM. Prevenção de lesões de pele no perioperatório: revisão integrativa da literatura. Rev Latino-am Enfermagem. 2006; 14(1):124-31.

10. Galvão CM, Sawada NO, Mendes IAC. A busca das melhores evidências.
Rev Esc Enferm USP. 2003; 37(4): 43-50.

11. Mangusan RF, Hooper V, Travis L. Outcomes associated with postoperative delirium after cardiac surgery. Am J Crit Care. 2015; 24 (2): 156-163.

12. Theologou S, Giakoumidaki K, Charitos C. Perioperative predictors of delirium and incidence factors in adult patients post cardiac surgery. Pragmat 0 bs Res. 2018; 9: 11-19.

13. Marra A, Ely EW, Pandharipande PP, Patel MB. The ABCDEF Bundle in Critical Care. Crit Care Clin. 2017 abr.; 33(2): 225-243.

14. Babadi ME, Shokrollahi N, Mehrabi T. Effect of family-patient communication on the incidence of delirium. Iran J Nurs Midwifery Res. 2017 jul/aug 22 (4): 327-31.

15. Oliveira KP, Picanço CM, Oliveira AR, Assis YIS, Souza ACF, Ribeiro AG. Estratégias utilizadas por enfermeiras para minimizar a ocorrência de delirium em pacientes críticos. Rev. Enferm. UFSM. 2020; 10:1-18.

16. Eberle CC, Santos AA, Macedo Junior LJJ, Martins JB. 0 Manejo Não Farmacológico do Delirium Sob a Ótica de Enfermeiros de uma Unidade de Terapia Intensiva Adulto. J. res. fundam. care. Online. 2019 out/dez; 11(5):1242-1249. 17. Koster S, Hensens AG, Oosterveld FGJ, Wijma A, Palen JVD. The delirium observation screening scale recognizes delirium early after cardiac surgery. Eur. J. Cardiovasc. 2009; 8:309-314.

18. Lingehall HC, Smulter N, Engstrom KG, Gustafson Y, Olofsson B. Validation of the Swedish version of the Nursing Delirium Screening Scale used in patients 70 years and older undergoing cardiac surgery. J. Clin. Nurs. 2013; 22:2858-2866.

19. Peréz AA, Poza SC, Catalán CC, Fernández AG, Vidal YJN. Valoración de la concordancia entre la escala CAM-ICU y la nursing delirium screening scale en el postoperatorio de cirugía cardiaca en una unidad de críticos. Enferm intensiva. 2014; 25(3): 100-106 